\title{
eJRIEPS
}

Ejournal de la recherche sur l'intervention en éducation physique et sport

Numéro spécial 1 | 2018

Comment penser les articulations entre approches didactiques et ergonomiques pour étudier les pratiques d'enseignement et de formation?

\section{Analyser l'activité évaluative des enseignants d'EPS : les apports des approches ergonomique et didactique}

Analyze the evaluative activity of the teachers of physical education: the contributions of the ergonomic and didactic approaches

Vincent Grosstephan et Stéphane Brau-Antony

\section{OpenEdition}

Édition électronique

URL : https://journals.openedition.org/ejrieps/358

DOI : $10.4000 /$ ejrieps.358

ISSN : 2105-0821

Éditeur

ELLIADD

Référence électronique

Vincent Grosstephan et Stéphane Brau-Antony, «Analyser l'activité évaluative des enseignants d'EPS : les apports des approches ergonomique et didactique », eJRIEPS [En ligne], Numéro spécial 1 | 2018 mis en ligne le 01 avril 2018, consulté le 21 septembre 2021. URL : http://journals.openedition.org/ ejrieps/358 ; DOI : https://doi.org/10.4000/ejrieps.358

La revue eJRIEPS est mise à disposition selon les termes de la Creative Commons Attribution 4.0 International License. 
eJRIEPS Numéro Spécial n¹ Avril 2018

Analyser l'activité évaluative des enseignants d'EPS :

les apports des approches ergonomique et didactique

Vincent Grosstephan* et Stéphane Brau-Antony*

* CEREP, EA4692, Université de Reims Champagne Ardenne, France.

Résumé

A travers l'évocation de leurs travaux sur les pratiques des enseignants d'EPS (enseignement et évaluation), et leur dynamique de développement les auteurs mobilisent de façon croisée les approches didactique et ergonomique de l'activité. Cet article présente un dispositif de recherche-formation portant sur l'évaluation aux examens d'EPS. Ce dispositif a pour objectif d'améliorer l'analyse et la compréhension des décisions des enseignants et notamment de montrer de quelles façons ils s'emparent des prescriptions diverses auxquels ils sont soumis pour exercer au mieux leur activité d'évaluation. Les résultats mettent en évidence l'intérêt de combiner les analyses ergonomique et didactique pour tenter de comprendre les déterminants de l'acte d'évaluation que l'on ne peut réduire au rapport que les enseignants entretiennent aux différentes sources de prescriptions.

Mots clés : certification, conceptions, didactique, ergonomie, évaluateur, prescription

\section{Introduction}

L'article présente un dispositif de recherche-formation associant dix enseignants d'Education Physique et Sportive (EPS) et deux enseignants-chercheurs ayant le statut de formateurs. Ce dispositif répond à une commande du Syndicat National de l'Education Physique, elle porte sur les pratiques d'évaluation aux examens et plus spécifiquement sur l'évaluation au Diplôme National du Brevet suite à l'instauration de nouvelles modalités d'évaluation en 2012. La réforme de l'évaluation en EPS au collège, sur le modèle des épreuves du baccalauréat, s'appuie sur des référentiels nationaux spécifiant les compétences à évaluer, l'épreuve d'évaluation, les critères et indicateurs ainsi que des repères de notation. L'enseignant a donc pour mission de noter les prestations des élèves à partir de ces référentiels institutionnels qui font office de prescriptions. La commande 


\section{eJRIEPS Numéro Spécial n¹ Avril 2018}

vise à répondre à une insatisfaction praxéologique manifestée par les enseignants à propos des référentiels d'évaluation qu'ils estiment inadaptés aux conditions réelles d'exercice du métier : contenu des référentiels et difficultés d'utilisation.

Le dispositif de recherche-formation poursuit conjointement deux visées :

- une visée épistémique dont l'objectif est de décrire et comprendre les déterminants et les caractéristiques de l'activité des évaluateurs et notamment l'usage qu'ils font des prescriptions qui leur sont adressées ;

- une visée transformative dont la finalité est de produire du développement chez les enseignants afin d'accroître leur expertise professionnelle. Le dispositif dont nous rendons compte est fondé sur l'analyse de situations de travail, l'objectif étant de favoriser chez les enseignants une compréhension de leur activité professionnelle en vue de se développer (Vinatier, 2012). Ce type de dispositif s'appuie sur les principes de l'apprentissage expérentiel à savoir un apprentissage qui passe par l'exercice d'une pensée réflexive du sujet sur sa propre pratique (Bourgeois et Nizet, 2005). Dans ce dispositif il s'agit de confronter sa pratique d'évaluation à d'autres points de vue de manière à mettre au jour ce qui fonde son jugement professionnel et en cas de désaccord avec ses pairs de développer une distance critique ouvrant la possibilité d'une réflexion sur ses manières habituelles de pratiquer l'évaluation. Le dispositif repose également sur l'idée qu'aider les enseignants à analyser leur propre expérience de travail et celle de leurs pairs nécessite de mobiliser un certain nombre de ressources ou d'apports externes mis à disposition par les formateurs. Certains auteurs font l'hypothèse que les savoirs produits par la recherche sur le travail enseignant constituent une ressource à privilégier pour les praticiens afin qu'ils puissent mieux décrire et comprendre leurs situations ordinaires de travail (Grosstephan et Brau-Antony, 2016 ; Perrenoud et al., 2008). Dans le dispositif que nous présentons les enseignants font ainsi l'expérience de l'analyse de leur activité d'évaluateur en la confrontant à celle de leurs pairs grâce aux apports externes des formateurs qui leur fournissent des outils conceptuels permettant de mieux saisir le sens de leur travail, dans ses dimensions visible et invisible, quand ils évaluent les productions des élèves à l'aide des référentiels.

Dans un premier temps nous aborderons l'ancrage théorique pilotant le choix du dispositif de recherche-formation en convoquant l'ergonomie de l'activité qui consiste notamment à identifier les différentes sources de prescriptions affectant le travail enseignant (Saujat et Serres, 2015) ainsi que le rôle qu'elles jouent dans le cadre des pratiques d'évaluation aux examens d'EPS et notamment la façon dont ces prescriptions sont reconfigurées par 


\section{eJRIEPS Numéro Spécial n¹ Avril 2018}

les enseignants (Brau-Antony et Hubert, 2014). Dans un second temps nous décrirons le dispositif de recherche-formation en nous arrêtant sur une situation de formation particulière au cours de laquelle les dix enseignants ont été amenés à noter des productions d'élèves restituées par un enregistrement vidéo. L'analyse des échanges initiée par une comparaison des notes délivrées par chacun des évaluateurs nous permettra de montrer que les débats ou controverses suscités par cette situation nécessitent de mobiliser d'autres concepts que ceux utilisés par l'ergonomie pour comprendre d'une part les déterminismes qui pèsent sur le modèle de référence des évaluateurs (Noizet et Caverni, 1978) et, d'autre part, analyser le rapport que les enseignants entretiennent aux prescriptions institutionnelles. Ainsi une approche didactique nous apparaît utile pour étudier comment s'opère le jugement de l'évaluateur, la démarche d'évaluation certificative ne pouvant être déconnectée des choix didactiques de l'enseignant et des conceptions de l'enseignement des pratiques physiques sportives et artistiques qui les sous-tendent.

\section{Cadre théorique : une approche ergonomique du travail enseignant}

Le dispositif de recherche-formation nous a amenés à positionner l'approche ergonomique comme première et l'approche didactique comme seconde. Nous optons pour une analyse du travail de l'évaluateur avec une entrée privilégiée qui est celle de l'ergonomie de langue française, dite encore "ergonomie de l'activité " (Hubault et Bourgeois, 2004). Pour autant, l'objet même de l'activité étudiée, à savoir l'évaluation aux examens, ne peut selon nous, faire l'impasse d'une réflexion sur sa dimension didactique. Bain (1988) et Chevallard (1986) soulignent en effet que l'évaluation n'est pas un phénomène autonome que l'on peut dissocier de l'ensemble du fonctionnement didactique. Certifier les résultats des apprentissages nécessite de vérifier que les élèves maîtrisent les exigences fixées par des programmes d'enseignement (De Ketele, 2001), les épreuves d'évaluation s'articulant aux contenus enseignés. En cela, l'approche didactique que nous mobilisons, vient " outiller » l'analyse du travail en lui fournissant notamment les concepts lui permettant de documenter de façon plus précise et plus spécifique les écarts entre le prescrit et le réalisé. Si l'approche didactique est ici seconde, elle n'est en rien secondaire et occupe, dans l'approche ergonomique, un statut de discipline contributive à l'analyse de l'activité, au regard de la spécificité du travail étudié. C'est donc au moment de donner du sens aux écarts entre le travail prescrit, le travail réalisé et le travail réel que nous utiliserons les apports des approches didactiques. 


\section{eJRIEPS Numéro Spécial n¹ Avril 2018}

Le travail que nous avons réalisé revendique donc la double finalité de l'approche ergonomique de l'activité : à la fois décrire et comprendre ce que fait l'opérateur (en l'occurrence l'enseignant qui évalue aux examens en EPS), mais également contribuer à la transformation de cette activité pour permettre aux professionnels de trouver les moyens de dépasser les tensions inhérentes à celle-ci.

\subsection{Analyser le travail pour décrire et comprendre}

Nous proposons une analyse du travail de l'évaluateur permettant d'accéder à une meilleure compréhension de ce qui nous semble le caractériser : la façon dont ce dernier gère les tensions entre les attentes de l'institution relatives aux modalités d'évaluation aux examens (les référentiels nationaux d'évaluation que nous qualifierons de prescriptions descendantes (Six, 1999), et les autres sources de prescription relatives à la situation de travail elle-même et au sujet enseignant. Six (1999) parle ici de prescriptions remontantes provenant de trois sources principales:

- la première est constituée du collectif de travail auquel appartient l'enseignant. Ce collectif, en discutant, re-travaillant, mettant à l'épreuve les prescriptions descendantes, fixe à l'enseignant de nouvelles attentes dont les visées peuvent être diverses: harmoniser les pratiques au sein de l'établissement dans un but d'égalité de traitement des élèves des différentes classes, faciliter l'utilisation de référentiels considérés comme peu opératoires pris tels quels comme c'est le cas pour les équipes pédagogiques en EPS qui n'hésitent pas à procéder à des arrangements par rapport aux prescriptions officielles qui leur sont adressées (Brau-Antony et Hubert, 2014). Saujat et Serres (2015, p. 7) qualifient ainsi ce travail de «première mise à l'épreuve des prescriptions descendantes en vue de les digérer »;

- la seconde est liée à la confrontation au travail réel en classe qui peut être considérée comme une autre source de prescription remontante. En effet, une des spécificités de l'évaluation aux examens en EPS consiste dans le fait que l'un des deux enseignants évaluateurs est celui qui a la charge de la classe durant l'année et dont la connaissance préalable des élèves peut constituer un déterminant non négligeable de ses décisions évaluatives, au même titre que la non connaissance de ces élèves peut provoquer un autre type de déterminations chez l'enseignant n'ayant pas en charge la classe ;

- la troisième source de prescription relève des auto-prescriptions de l'enseignant qui personnalise les prescriptions exogènes qui lui sont destinées. Nous faisons l'hypothèse que ces auto-prescriptions constituent une actualisation de la façon dont l'enseignant tente de situer et de rendre acceptable son activité d'évaluateur au milieu de cet 


\section{eJRIEPS Numéro Spécial n¹ Avril 2018}

ensemble de prescriptions. De fait, il est amené à effectuer des choix, à envisager des compromis entre ce qu'il pense devoir faire et ce qu'il pense possible de faire. Nous considérons donc que l'activité de l'évaluateur résulte d'une confrontation, parfois d'une lutte entre des déterminants externes et des déterminants internes relatifs au rapport subjectif de l'enseignant à la tâche que l'institution lui assigne, dans les conditions situées dans lesquelles il doit la réaliser: noter les prestations des élèves sur une séance d'évaluation terminale qui prolonge le travail en classe durant le cycle d'enseignement.

2.2. Intervenir pour transformer l'activité

II s'agit ici plus particulièrement de s'interroger sur les conditions d'intervention en analyse du travail susceptibles de permettre une meilleure gestion des tensions évoquées plus haut et par là-même un développement de l'activité. Nous en retiendrons trois principales. La première est celle de s'astreindre à une analyse précise des gestes quotidiens de travail (Quillerou-Grivot et Clot, 2013). La seconde renvoie au rôle du collectif dans le développement individuel. En considérant que "l'individuel chez l'homme n'est pas le contraire du social mais sa forme supérieure ", Vygotski (1929, p. 236), pose les bases de sa conception du rôle du collectif dans le développement du sujet. "L'individu devient sujet psychologique quand il se met à employer à son propre égard et à sa manière propre, unique en son genre, les formes de conduite que les autres ont employées d'abord envers lui » ajoute Clot (2015, p. 210). Le collectif devient dès lors une ressource pour l'individu, non pas que ce dernier en soit le simple produit, mais par le double processus d'affranchissement à l'égard des contraintes génériques et de sa propre histoire que le sujet opère grâce à lui (Clot, Faïta, Fernandez et Scheller, 2001). Vygotski nous permet enfin d'évoquer la troisième condition qui est de donner un sens à la prise de conscience de ses expériences vécues que le sujet peut réaliser dans le cadre des méthodologies déployées dans le dispositif. II souligne ainsi qu' " avoir conscience de ses expériences vécues n'est rien d'autre que les avoir à sa disposition à titre d'objet pour d'autres expériences vécues. La conscience est l'expérience vécue d'expériences vécues » (1924, p. 78). Selon l'auteur, l'expérience passée devient ainsi un moyen pour vivre la situation présente ou la situation future notamment quand la prise de conscience permet de redécouvrir l'incomplétude du sujet à travers le fait que l'activité passée était pleine de possibilités non réalisées, rendant ainsi le sujet disponible au développement.

Cependant nous n'évoquons la finalité de transformation, que pour faciliter la compréhension du dispositif et du contexte particulier au sein duquel nous tentons d'approcher l'activité d'évaluation des enseignants. Nous ne documenterons donc pas les 
eJRIEPS Numéro Spécial n¹ Avril 2018

effets produits par ce dernier en termes de développement sur les enseignants participants.

Cet article vise ainsi à mettre au jour trois principales dimensions de l'activité d'évaluation aux examens d'enseignants d'EPS :

- celle de la plus ou moins grande prise de distance avec les prescriptions institutionnelles et des mobiles qui en sont à l'origine ;

- celle de la dimension didactique du processus évaluatif ;

- celle relative au poids des conceptions de l'EPS et de la pratique physique sportive et artistique (PPSA) enseignée chez les enseignants.

\section{Méthodologie}

3.1. Présentation de la situation de formation et recueil de données

Les dix enseignants participant à la formation ont entre 10 et 18 ans d'expérience d'enseignement et proviennent de collèges différents. La situation de formation à laquelle ils sont confrontés possède un air de famille avec l'activité ordinaire des évaluateurs. Elle vise à reproduire en partie les caractéristiques du travail de l'enseignant lorsqu'il est amené en fin de cycle d'apprentissage à évaluer les productions des élèves en utilisant le référentiel de certification. Cette situation de formation peut être considérée comme une situation potentielle de développement (Mayen, 1999) dans la mesure où elle comporte un problème à résoudre et à construire et prévoie un moment de débriefing où l'activité réflexive des participants est sollicitée.

La tâche à réaliser était la suivante : les dix enseignants et les deux formateurs devaient noter les productions de cinq élèves de troisième de collège en boxe française à partir d'un enregistrement vidéo fourni par l'enseignant responsable de la classe. La sélection des élèves s'est faite sur proposition de ce dernier. II s'agissait de proposer un panel le plus représentatif possible des différents niveaux présents au sein de la classe. Cette notation a été effectuée à partir du référentiel national (cf. annexe) et ne portait pas sur l'ensemble des items. En effet afin de simplifier la prise d'information et de la centrer sur les prestations motrices des élèves, les rôles d'arbitre, de juge, d'observateur et de conseiller, notés sur 4 points, ainsi que le gain des assauts, noté sur 2 points, n'ont pas été évalués. La notation s'appuyait ainsi sur les items suivants : efficacité dans le gain de l'assaut (sur 6 points) et efficacité du tireur dans ses enchaînements (sur 8 points). Ce second critère sert de référent pour l'évaluation des prestations des élèves dans une 


\section{eJRIEPS Numéro Spécial n¹ Avril 2018}

situation de duo qui constitue une épreuve d'évaluation non prévue par le référentiel : les élèves sont dans ce cas en situation de coopération et non pas d'affrontement tel que le prescrit le référentiel. Un seul visionnage de la vidéo a été proposé aux évaluateurs de manière à se rapprocher de la situation de travail habituelle au cours de laquelle l'enseignant saisit sur le vif un certain nombre d'indices sur les réalisations des élèves afin de porter une appréciation sur leur niveau de compétence. Les traces de l'activité des élèves dans la situation d'évaluation contrairement aux « empreintes » laissées sur une copie sur laquelle il est possible de revenir sont donc très éphémères. L'enseignant responsable de la classe a souligné à ce propos "qu'évaluer cinq élèves en même temps, c'est déjà énorme (trois duels en même temps) ».

Trois filles et deux garçons ont été évalués. Le relevé et une analyse rapide des notes (étendue, moyenne, ordre) a ensuite permis de pointer un certain nombre de convergences et de divergences entre évaluateurs : ordres différents des notes selon les évaluateurs, écarts significatifs de notes sur certains élèves en particulier. Les divergences ont été ensuite soumises à discussion entre les enseignants de façon à tenter d'en identifier les raisons.

Les données produites lors de cette situation de formation sont donc de deux ordres : des notes proposées par les enseignants et des enregistrements de discussions et débats relatifs aux divergences de notation identifiées. Le second type de données correspond aux données principales de notre étude, le premier type de données jouant principalement le rôle de support et de prétexte au débat. En effet, il ne s'agit pas de montrer que des divergences existent, cela a été depuis longtemps établi par les travaux en docimologie (Piéron, 1963). Si le faible nombre de notes ne permet pas d'en faire une exploitation statistique significative il donne l'occasion néanmoins de pointer des divergences significatives à propos desquelles les arguments déployés par les enseignants constituent des informations utiles pour comprendre l'activité des évaluateurs.

\subsection{Traitement des données}

Les échanges entre enseignants et formateurs lors de l'analyse collective des notes ont été enregistrés et transcrits verbatim. Le traitement des données s'est déroulé en trois temps (cf tableau 1): 


\section{eJRIEPS Numéro Spécial n¹ Avril 2018}

- le premier temps a consisté à identifier les objets de discours (ce dont on parle) ainsi qu'à repérer comment un objet de discours peut se trouver reconverti en objet de débat (confrontation de points de vue divergents sur cet objet), les enseignants étant incités à argumenter et échanger leurs points de vue ;

- le second temps a cherché à documenter les différentes sources de prescriptions (prescriptions descendantes et prescriptions remontantes) évoquées par les enseignants lors des débats ;

- le troisième temps visait à identifier le contenu de ces sources de prescriptions afin d'en faire une analyse didactique.

Tableau 1. Exemple de traitement des données.

\begin{tabular}{|c|c|c|c|}
\hline Objet de discours & Objet de débat & $\begin{array}{c}\text { Sources } \\
\text { prescriptions }\end{array}$ & $\begin{array}{l}\text { Analyse } \\
\text { didactique } \\
\text { prescriptions }\end{array}$ \\
\hline $\begin{array}{l}\quad \text { Choix du duo } \\
\text { comme épreuve } \\
\text { d'évaluation pour } \\
\text { évaluer l'efficacité } \\
\text { du tireur dans ses } \\
\text { enchaînements } \\
\text { (élément à évaluer } \\
\text { du référentiel } \\
\text { officiel) }\end{array}$ & $\begin{array}{l}\text { - L'épreuve } \\
\text { d'évaluation n'est } \\
\text { pas conforme à } \\
\text { l'épreuve officielle } \\
\text { (le duel) } \\
\text { - Le duo est une } \\
\text { épreuve } \\
\text { d'évaluation qui vise } \\
\text { principalement à } \\
\text { rendre compte de ce } \\
\text { qui a été enseigné } \\
\text { en cours de cycle } \\
\text { mais ce qui est } \\
\text { enseigné } \\
\text { contesté est } \\
\text { (conceptions } \\
\text { divergentes de la } \\
\text { boxe française) }\end{array}$ & 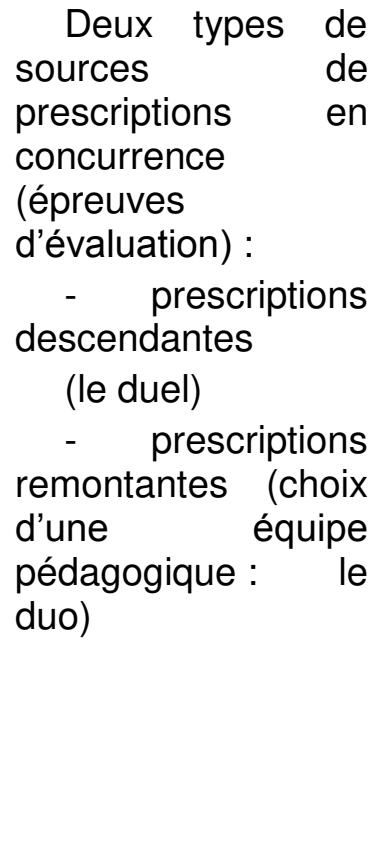 & $\begin{array}{l}\text { - Le duo renvoie } \\
\text { à des choix } \\
\text { didactiques, une } \\
\text { constitue } \\
\text { situation } \\
\text { d'apprentissage qui } \\
\text { balise le cycle. II } \\
\text { s'agit de vérifier la } \\
\text { maîtrise par les } \\
\text { élèves de ce qui a } \\
\text { été enseigné } \\
\quad \text { - Le duo fait } \\
\text { partie de la logique } \\
\text { des sports de } \\
\text { combat et favorise } \\
\text { l'apprentissage de } \\
\text { certaines techniques } \\
\text { considérées comme } \\
\text { essentielles }\end{array}$ \\
\hline
\end{tabular}

\section{Résultats : l'évaluation une activité à forte composante didactique}

Comme nous l'avons évoqué précédemment, l'épreuve d'évaluation à laquelle sont soumis les élèves ne correspond pas à la prescription institutionnelle qui vise à proposer des assauts de deux à trois minutes de plusieurs rounds. Celle à laquelle sont effectivement confrontés les élèves de la classe est une épreuve de duo dont les enchaînements sont préparés à l'avance. L'analyse de cette différence essentielle entre 


\section{eJRIEPS Numéro Spécial n¹ Avril 2018}

l'épreuve prescrite et l'épreuve réalisée et les débats qui en résultent nous permettent de relever un élément clé du processus d'évaluation : le poids des prescriptions remontantes et de leurs fortes dimensions didactique et technologique.

\subsection{Les prescriptions remontant du collectif}

L'équipe pédagogique à laquelle appartient l'enseignant responsable de la classe a opéré un premier travail d'adaptation du référentiel de façon à le rendre utilisable par les enseignants de l'équipe. Cette adaptation porte sur deux éléments : l'évaluation de l'item efficacité du tireur dans ses enchaînements se fait dans une épreuve de duo et non de duel comme nous le précisions plus haut. Par ailleurs, un certain nombre d'indicateurs du référentiel sont reformulés voire totalement modifiés. L'équipe de l'établissement a estimé que l'inadaptation de l'épreuve à la réalité des conditions de pratiques, au niveau des élèves et à leurs conséquences sur les effets de l'EPS sur la pratique ultérieure d'une activité physique, justifiait une adaptation de l'épreuve. L'équipe a ainsi tranché le dilemme, réapparu dans les échanges entre enseignants : faut-il privilégier l'adaptation au réel au bénéfice des élèves de l'établissement ou faut-il préserver le souci d'égalité de traitement entre élèves de l'ensemble des établissements en maintenant la même épreuve pour tous et en tous lieux? Le dilemme est tranché et exprimé ainsi par un enseignant : "si on note avec les critères du référentiel dans une situation de duel, ils ont tous entre 0 et 7. Or, l'enjeu de l'EPS c'est de donner envie aux élèves de pratiquer en dehors de l'école. Si on ne met que des mauvaises notes on les démotive ".

Outre l'adaptation de l'épreuve, les enseignants de cet établissement proposent également une adaptation des indicateurs du référentiel, soit sous forme de reformulation, ainsi l'indicateur grand volume d'enchaînements avec une efficacité relative pour marquer des points devient "cherche à reprendre l'avantage sur l'adversaire et quelques enchaînements", soit en en changeant radicalement la nature, par exemple l'item efficacité réelle des enchaînements pour marquer des points est remplacé par « reste au centre $d u$ ring ». Ces résultats soulignent ainsi les écarts entre le travail prescrit et le travail réalisé.

\subsection{L'épistémologie de l'évaluateur}

\subsubsection{L'évaluation : un fait didactique}

L'enseignant responsable de la classe déploie un premier argument montrant que l'évaluation terminale doit nécessairement porter sur ce qui a été enseigné : «j'évalue ce que j'ai fait ». Comme le souligne Raisky (1996) les épreuves d'évaluation ponctuelles 


\section{eJRIEPS Numéro Spécial n¹ Avril 2018}

dépendent fortement des contenus enseignés, l'évaluation est à l'interface du travail de l'enseignant (quels sont les savoirs qui méritent d'être enseignés compte tenu des contraintes du temps didactique ?) et du travail de l'élève (quels sont les savoirs qui sont assimilés ?). C'est donc dans cette perspective que se situe cet enseignant qui justifie ainsi ses choix didactiques en considérant que la touche à la tête, dont il est dit dans le référentiel qu'elle peut être interdite, est un "apprentissage central» [...] " dans la logique de l'activité ». La touche pied/tête est, selon lui, abordable en duo mais pas en duel pour des questions de sécurité, de maîtrise limitée des élèves, de problèmes de contrôle de la touche par "manque de souplesse quand la jambe elle part " [...] " sans protection je fais pas ». Cet apprentissage est au cœur du contrat didactique (Brousseau, 1990) structuré autour des intentions didactiques de l'enseignant et de l'agencement des situations d'apprentissage proposées aux élèves. La situation en duo constitue le fil conducteur des séances, les élèves y sont confrontés plusieurs fois au cours du cycle: "si j'évalue les enchaînements en assaut je verrouille, je ne propose plus les mêmes variétés techniques, du genre pieds à la tête, alors que je les ai travaillées en cours, et du coup je n'évalue plus ce que j'ai travaillé en cours ». L'évaluation des élèves est donc pilotée par des choix didactiques clairement assumés par cet enseignant. L'enseignant part ainsi du principe que la touche pied/tête est " un attendu » fondamental qui traduit sa conception de l'enseignement de la boxe française. II signale d'ailleurs que cela fait débat avec un collègue de son établissement qui l'autorise en duel. II précise ensuite ce qu'il a effectivement enseigné : “j'ai fait trois niveaux, chassé ... chassé et parade, chassé latéral ... et revers " [...] " après j'ai beaucoup travaillé sur les esquives, sur les blocages, sur les blocages en chassé » ce qui n'apparaît pas dans le référentiel. Le duo permet ainsi de mieux restituer au cours de l'évaluation la diversité des apprentissages effectués et notamment les techniques travaillées qui n'apparaîtraient pas en situation d'assaut «ce qui aboutirait à des notes catastrophiques ».

Ces différents contenus d'enseignement et donc d'évaluation sont à resituer dans le constat que fait cet enseignant d'un décalage important entre les critères du référentiel et ce que sont capables de faire ses élèves. Ils témoignent également de la nécessité de préciser les contenus à enseigner au regard de la formulation relativement générale de ces contenus dans le référentiel national, permettant de fait de nombreuses interprétations. Ce constat est partagé par les neuf enseignants : "le nombre de touches je l'ai estimé »; " je fais avec les moyens du bord, je traduis volume d'enchaînements par pas mal d'actions quelle que soit la pertinence des actions ». La prescription s'avère donc 
eJRIEPS Numéro Spécial n¹ Avril 2018

relativement floue et ne donne pas suffisamment d'indications sur les transformations visées ("c'est quoi un enchaînement simple ?").

Les arguments avancés par l'enseignant responsable de la classe font par ailleurs débat au point qu'un des enseignants refuse d'évaluer les prestations des élèves sous prétexte qu'il n'est pas acceptable que l'on change les règles du jeu en modifiant l'épreuve d'évaluation. Cet enseignant ne conteste pas l'intention de l'enseignant responsable de la classe qui souhaite "s'adapter au profil des élèves, au public, l'assaut peut poser problème car l'investissement émotionnel est important » mais considère que l'on ne peut s'autoriser à transgresser les prescriptions officielles. Premièrement parce que le duo ne peut se substituer au duel en tant qu'épreuve d'évaluation et deuxièmement parce que les critères présents dans le référentiel ont été choisis pour évaluer les productions des élèves au cours de plusieurs assauts et non pas dans une situation de coopération. Par ailleurs, même s'ils ont joué le jeu de l'évaluation en notant les élèves, deux enseignants émettent des réserves à propos des choix didactiques conduisant à évaluer des compétences construites en cours du cycle en duo. Pour le premier, le duo est qualifié de " chorégraphie ", pour le second "c'est de la danse " et "c'est un peu loin de l'activité " ce qui peut expliquer la lenteur des enchaînements réalisés par les élèves qui reproduisent ce qui a été appris et répété en duo. Dans ce cas les désaccords ne portent pas sur la nécessité de respecter les exigences du référentiel mais sur le transfert de compétences construites en situation de coopération dans la situation d'opposition prescrite par le référentiel.

\subsubsection{Le poids des conceptions des enseignants}

Le second type d'argument concerne la conception que l'enseignant responsable de la classe a de l'enseignement de la boxe française, conception qui transparaît déjà à travers les contenus évoqués précédemment avec l'importance accordée au duo dans une activité d'opposition. Pour cet enseignant, " de toute façon on parlait de la logique de l'activité qui consiste à toucher à la tête mais dans tous les sports de combat la logique de duo elle existe " faisant valoir son expérience personnelle de pratiquant, spécialiste de judo. II introduit ainsi une dimension de la pratique sociale de référence (Martinand, 1987) qu'il considère comme légitime à enseigner et à évaluer.

Par ailleurs, lorsque l'on compare les indicateurs évoqués lors des débats par les différents enseignants pour justifier leurs notes on constate de fortes disparités que nous attribuons à des niveaux de connaissance des pratiques physiques sportives et artistiques très hétérogènes. Ces connaissances technologiques renvoient à la spécificité 
eJRIEPS Numéro Spécial n¹ Avril 2018

et à l'essence des PPSA et aux valeurs qui leur sont associées (Bouthier, 2008). Mobilisables par les enseignants elles permettent d'une part d'examiner les fondements de la pratique sociale prise pour référence et d'autre part de déterminer la pratique scolaire visée : comment transposer la culture sportive et artistique pour qu'elle puisse être enseignable à l'école ? Ces connaissances constituent donc des ressources pour l'intervention, elles permettent de décrire, analyser mais aussi évaluer les productions des élèves.

Prenons l'exemple d'un élève dont les prestations ont été diversement appréciées par les enseignants, les indicateurs retenus par les uns et les autres faisant débat. Cette diversité d'indicateurs se caractérise à la fois par son côté hétéroclite ( c c'est le seul qui a fait une touche du pied »; "il fait des enchaînements adaptés »; "il n’a fait qu'une touche adaptée »; "c'est le seul qui avait quelqu'un qui attaquait en face »...) mais également par des points de vue radicalement opposés sur ce qui atteste une réelle efficience : pour certains enseignants, ce qui est caractéristique de l'efficience est un grand volume d'activité et de déplacements alors que l'enseignant responsable de la classe spécialiste de sports de combat considère que « celui qui est au centre du ring c'est celui qui domine le combat ». Le volume d'activité n'est donc pas, selon lui, un argument pertinent, bien au contraire pour juger le niveau de compétence d'un élève en boxe française.

Ces résultats nous permettent également de pointer des épistémologies pratiques d'évaluateur à l'œuvre quand les enseignants sont amenés à évaluer des prestations d'élèves et sont conviés à échanger sur le choix de leurs notes. Comme l'indiquent Tourmen et Mayen (2012, p. 71) «les évaluateurs mettent en place les conditions pour assurer leurs jugements et, parfois, réfréner leur propre tendance à juger trop vite. En effet, les référents expérentiels mobilisés par les évaluateurs ne concernent pas uniquement les phénomènes évalués, mais également les manières d'évaluer, de porter des jugements, et donc de connaître et d'apprécier la valeur des phénomènes en jeu. Les évaluateurs mobilisent ainsi leur expérience d'autres situations d'évaluation, c'est-à-dire de situations où ils ont pu faire l'expérience des phénomènes ». On pourrait donc considérer l'épistémologie de l'évaluateur comme une théorie d'action à usage professionnel qui a des conséquences pratiques sur l'activité de l'évaluateur et notamment sur les décisions qui orientent son jugement professionnel (Bressoux et Pansu, 2003 ; Mottier Lopez et Allal, 2010). Les résultats mettent en évidence que cette épistémologie pratique concerne: le choix des objets d'évaluation en fonction des contenus enseignés, la détermination de l'épreuve d'évaluation, la sélection de critères et 


\section{eJRIEPS Numéro Spécial n¹ Avril 2018}

d'indicateurs en lien avec sa conception des sports de combat et de son enseignement, les acquisitions possibles des élèves au regard de la durée des apprentissages scolaires et plus largement leur expérience d'évaluateur construite au fil du temps dans des contextes d'enseignement divers et variés ainsi que leur expérience de pratiquant de la PPSA enseignée (Loizon et Carnus, 2014).

\section{Conclusion}

Les résultats présentés montrent que le recours à l'approche didactique nous semble pertinent pour nourrir l'analyse ergonomique de l'activité évaluative des enseignants, saisir les ressorts du jugement des évaluateurs et identifier, par là-même, le contenu des arguments avancés par les enseignants pour justifier leurs notes. Les résultats mettent en évidence l'intérêt de combiner les analyses ergonomique et didactique pour tenter de comprendre les déterminants de l'acte d'évaluation. En documentant précisément les dimensions relatives aux contenus enseignés et à leur évaluation, l'approche didactique permet d'appréhender de façon plus spécifique l'objet de l'activité étudiée : l'évaluation des élèves en EPS aux examens. La teneur des débats entre les enseignants souligne en effet que l'évaluation certificative est un phénomène didactique à part entière. Si le but visé par les évaluateurs consiste à répondre à ce qu'on leur demande de faire c'est-à-dire mettre une note à chaque élève à partir d'un référentiel, restent que les préoccupations didactiques sont très présentes quand on analyse le contenu des arguments avancés par les enseignants dans la situation de formation que nous avons décrite.

Par ailleurs, le dispositif sur lequel nous nous sommes appuyés nous semble devoir être interrogé. Travailler «avec» des praticiens engage le chercheur dans une dimension éthique et idéologique de son rapport avec eux. Les praticiens ne peuvent avoir pour seul statut ceux d'objets ou de moyens de la recherche. Le chercheur s'engage à être au service du développement, de l'émancipation, de la réflexivité des professionnels (Schön, 1994), du développement de leur pouvoir d'agir (Clot, 1999). La visée épistémique ne peut se réaliser au détriment de cet engagement. De même les praticiens s'engagent à donner à voir leurs pratiques, le sens qu'ils y construisent et à accepter la confrontation des analyses et interprétations. Ce postulat de «compétence réflexive » est au fondement de la possibilité de faire de la recherche « avec » des praticiens et l'intensité et la persistance de leur engagement semblent dès lors essentielles. C'était un des enjeux majeurs de ce dispositif. 


\section{eJRIEPS Numéro Spécial n¹ Avril 2018}

Pour autant, l'articulation de visées différentes (épistémiques et formatives) ne peut pas reposer uniquement sur une éthique de la relation. Elle suppose également un cadre épistémologique et théorique pour penser et fonder la relation au service des différents types d'objectifs. Comme le soulignent Vinatier et Morrissette (2015, p. 157) : « on voit bien que ce qui est en question c'est de ne pas confondre l'activité du chercheur qui « fait de la recherche " et celle du praticien qui " est en recherche ", alors que l'un et l'autre se mobilisent conjointement sur un problème commun ». La référence fondatrice aux travaux de Dewey (2011) et le développement plus récent des théories de l'activité, dans leur diversité mais aussi dans leur unité (Barbier et Durand, 2003 ; Albero et Guérin, 2014) constituent dès lors des points d'appuis essentiels.

Enfin, comme nous l'avons signalé plus haut, nous pensons que le rôle du chercheurformateur ne peut se limiter à organiser les controverses entre enseignants sur leur activité mais qu'il doit aussi instrumenter l'analyse de leurs pratiques par des apports de ressources telles que les travaux de recherche sur le travail enseignant.

\section{Bibliographie}

Albero, B. \& Guérin, J. (2014). L'intérêt pour « l'activité » en sciences de l'éducation. Vers une épistémologie fédératrice ? Note de synthèse. TransFormations, 11, 11-45.

Bain, D. (1988). Pour une formation à l'évaluation intégrée à la didactique. In M. GatherThurler \& P. Perrenoud, Savoir évaluer pour mieux enseigner : quelle formation des maîtres? (pp. 21-37). Service de la recherche sociologique de Genève, Cahier $n^{\circ} 26$.

Barbier, J.-M. \& Durand, M. (2003). L'activité : un objet intégrateur pour les sciences sociales ? Recherche et Formation, 42, 99-117.

Bourgeois, E. \& Nizet, J. (2005). Apprentissage et formation d'adultes. Paris : PUF.

Bouthier, D. (2008). Technologie des APSA : évolution des recherches et de leur place dans le cursus STAPS. eJRIEPS, 15, 44-59.

Brau-Antony, S. \& Hubert, B. (2014). Curriculum en Education Physique et Sportive et évaluation certificative au baccalauréat. Questions vives, 22, 67-82.

Bressoux, P. \& Pansu, P. (2003). Quand les enseignants jugent leurs élèves. Paris : PUF. Brousseau, G. (1990). Le contrat didactique: le milieu. Recherches en didactique des mathématiques, 9 (3), 309-336. 


\section{eJRIEPS Numéro Spécial n¹ Avril 2018}

Chevallard, Y. (1986). Pour une analyse didactique des faits d'évaluation. In J.-M. De Ketele, L'évaluation: approche descriptive ou prescriptive? (pp. 31-59). Bruxelles : De Boeck.

Clot, Y. (1999). La fonction psychologique du travail. Paris : PUF.

Clot, Y. (2015). Vygotski avec Spinoza, au-delà de Freud. Revue philosophique de la France et de l'étranger, 2/140, 205-224.

Clot, Y., Faïta, D., Fernandez, G. \& Scheller, L. (2001). Entretiens en autoconfrontation croisée : une méthode en clinique de l'activité. Education Permanente, 146, 1725.

De Ketele, J.-M. (2011). L'évaluation et le curriculum : les fondements conceptuels, les débats, les enjeux. Les Dossiers des Sciences de l'Education, 25, 89-106.

Dewey, J. (2011). Démocratie et éducation. Suivi de Expérience et éducation. Paris: Armand Colin.

Grosstephan, V. \& Brau-Antony, S. (2016). Faire parler des traces d'analyse d'une activité professionnelle : étude d'un processus d'argumentation. In T. Philippot, Les traces de l'activité. Objets pour la recherche et outils pour la formation (pp. 135-164). Paris : L'Harmattan. Action \& Savoir.

Hubault, F. \& Bourgeois, F. (2004). Disputes sur l'ergonomie de la tâche et de l'activité, ou la finalité de l'ergonomie en question, @ctivités, 1-1, 34-53.

Loizon, D. \& Carnus, M.-F. (2014). L'influence des déterminants personnels dans les choix didactiques des enseignants d'EPS. eJRIEPS, 33, 30-48.

Martinand, J.-L. (1987). Pratiques de référence, transposition didactique et savoirs professionnels. Les Sciences de l'éducation pour l'ère nouvelle, 1/2, 23-29.

Mayen, P. (1999). Des situations potentielles de développement. Recherche et Formation, 139, 65-86.

Mottier Lopez, L. \& Allal, L. (2010). Le jugement professionnel en évaluation : quelles triangulations méthodologiques et théoriques? In L. Paquay, C. Van Nieuwenhoven \& P. Wouters, L'évaluation, levier du développement professionnel. Tensions, dispositifs, perspective (pp. 237-249). Bruxelles: De Boeck.

Noizet, G. \& Caverni, J.-P. (1978). Psychologie de l'évaluation scolaire. Paris : PUF. 


\section{eJRIEPS Numéro Spécial n¹ Avril 2018}

Perrenoud, P., Altet, M., Lessard, C. \& Paquay, L. (2008). Conflits de savoirs en formation d'enseignants. Entre savoirs issus de la recherche et savoirs issus de l'expérience. Bruxelles : De Boeck.

Piéron, H. (1963). Examens et docimologie. Paris : PUF.

Quillerou-Grivot, E. \& Clot, Y. (2013). Trois conditions pour une clinique de l'activité en psychologie du travail : le cas d'une intervention dans une entreprise de logistique automobile. @ctivités, 10/2, 229-248.

Raisky, C. (1996). Doit-on en finir avec la transposition didactique ? In C. Raisky \& M. Caillot, Au-delà des didactiques, le didactique. Débats autour de concepts fédérateurs (pp. 37-59). Bruxelles : De Boeck.

Saujat, F. \& Serres, G. (2015). L'activité de l'enseignant d'EPS entre préoccupations et «occupations » : un point de vue développemental. eJRIEPS, 34, 4-30.

Schön, D. (1994). Le praticien réflexif. A la recherche du savoir caché dans l'agir professionnel. Montréal : Les Editions Logiques.

Six, F. (1999). De la prescription à la préparation du travail : apports de l'ergonomie à la prévention et à l'organisation du travail sur les chantiers du bâtiment. Note de synthèse pour l'habilitation à diriger des recherches, Université Charles de Gaulle Lille 3.

Tourmen, C. \& Mayen, P. (2012). Les évaluateurs, des épistémologues en actes ? In L. Mottier Lopez, L. \& G. Figari, Modélisations de l'évaluation en éducation (pp. 6377). Bruxelles : De Boeck.

Vinatier, I. (2012). Réflexivité et développement professionnel. Une orientation pour la formation. Toulouse : Octarès.

Vinatier, I. \& Morrissette, J. (2015). Rencontres entre chercheurs et praticiens : quels enjeux ? Carrefours de l'Education, 39, 137-170.

Vygotski L.-S. (1929/2004). Psychologie concrète de l'homme. In M. Brossard, Lectures et perspectives de recherche en éducation (pp. 225-255). Villeneuve d'Ascq: Septentrion. 